\title{
Stents Farmacológicos para Lesões em Bifurcações: Outra Indicação Não Aprovada (Off-Label) com Resultados Superiores aos dos Stents Não-Farmacológicos
}

\author{
Ver artigo relacionado \\ na página 406
}

\author{
Antonio Colombo ${ }^{1}$, Azeem Latib ${ }^{1}$
}

0 tratamento percutâneo das lesões em bifurcações coronárias continua sendo uma área de considerável interesse, não somente pela complexidade técnica durante o procedimento, mas também pelos índices historicamente elevados da necessidade de uma nova revascularização do vaso-alvo e de eventos cardíacos adversos em comparação às lesões simples $^{1}$. O surgimento dos stents farmacológicos (SF) e sua posterior aplicação em subgrupos de pacientes com lesões mais complexas pareceu ser uma solução plausível para redução da reestenose e da nova revascularização das lesões de bifurcação. Assim, no estudo ARTS II² (Arterial Revascularization Therapies Study parte II), os índices de eventos no primeiro ano foram semelhantes para lesões em bifurcações comparadas às lesões fora das bifurcações. Contudo, ao examinar estudos no "mundo real", não é incomum o relato de índices de dois dígitos de reestenose no acompanhamento a longo prazo $^{3}$. É importante lembrar que a maioria desses estudos reporta a reestenose angiográfica sem avaliação do impacto clínico real dessas lesões na geração de isquemia. O entusiasmo inicial pelos SF foi arrefecido pelo reconhecimento de risco tardio associado de trombose no stent (TS $)^{4}$. Esse risco não parece excessivo em lesões com indicações aprovadas, mas foram levantadas dúvidas sobre a segurança dos SF em lesões que não se enquadram nessas indicações. De fato, bifurcações foram consideradas uma das indicações não aprovadas para SF, as quais podem apresentar risco particularmente alto de $\mathrm{TS}^{4,5}$. Assim, em uma era em que eficácia e segurança dos SF em lesões sem indicação são questionadas, felicitamos os autores por nos oferecerem mais dados, principalmente os resultados a longo prazo.
Considerando a freqüência com que bifurcações são encontradas na prática diária intervencionista (15\% a $20 \%$ das intervenções coronárias percutâneas [ICP] executadas), é de certa forma surpreendente que não haja estudos randomizados direcionados para a comparação entre SF e stents não-farmacológicos (SNF) em bifurcações. Os únicos dados randomizados comparando SF e SNF resultam de uma subanálise do estudo SCANDSTENT ${ }^{6}$ (Stenting Coronary Arteries in Non-Stress/ Benestent Disease), que examinou um total de 126 pacientes com lesões em bifurcações tratadas com stents com eluição de sirolimus (SES) ou SNF. Em 55\% da coorte com SES e em 53\% da coorte com SNF, foram implantados stents em ambos os ramos da bifurcação. O implante de SES foi associado a significativa redução do índice de reestenose no ramo principal $(4,9 \%$ vs. $28,3 \% ; p<0,001)$ e no ramo lateral $(14,8 \%$ vs. $43,4 \% ; p<0,001)$, assim como a eventos cardíacos adversos maiores (ECAM) (9\% vs. 28\%; $p=0,009)$ durante os sete meses do período de acompanhamento ${ }^{1,6}$. Atualmente é improvável que se realize um estudo randomizado especificamente para comparar SF e SNF em bifurcações. A razão disso está nos índices muito altos de reestenose ocorrida após o implante de SNF em lesões em bifurcações, qualquer que seja a técnica usada, fazendo com que a realização de um estudo desse tipo seja quase "antiética".

Nesta edição da Revista Brasileira de Cardiologia Invasiva, Mendes et al. ${ }^{7}$ demonstram os resultados de uma comparação contemporânea, no "mundo real", entre SF e SNF em lesões coronárias em bifurcações. Os autores compararam 89 bifurcações tratadas com quatro tipos diferentes de $\mathrm{SF}$, em uma instituição, com 106 bifurcações tratadas com SNF variados, em outra

1 San Raffaele Scientific Institute e EMO-GVM Centro Cuore Columbus - Milão, Itália.

Correspondência: Antonio Colombo. EMO-GVM Centro Cuore Columbus. Via Buonarroti, 48 - Milano, Italia - 20145

E-mail: info@emocolumbus.it

Recebido em: 10/12/2008 • Aceito em: 12/12/2008 
instituição. A utilização do grupo de controle de SNF de outra instituição pode ser considerada uma limitação desse estudo, mas o fato de que os mesmos profissionais realizaram os procedimentos limita os vieses do tratamento. Embora torne mais difícil fazer afirmações sobre a eficácia de um SF específico, a inclusão de diferentes plataformas de SF reflete a prática diária. Os grupos SF e SNF foram bem pareados quanto a características clínico-angiográficas e do procedimento e aproximadamente três quartos das lesões eram bifurcações verdadeiras (ou seja, lesão > 50\% nos dois ramos). A estratégia provisional (implantar stent somente no ramo principal e angioplastia com balão no ramo lateral) foi utilizada na maioria das bifurcações (SF 75\% vs. SNF 89\%), e, embora não significativa estatisticamente, foram implantados stents nos dois ramos das bifurcações, com freqüência duas vezes maior com SF (25\%) que com SNF (11\%). Em 24 meses de acompanhamento clínico, os SF foram associados a reduções significativas da revascularização da lesão-alvo (RLA) $(2,2 \%$ vs. $18 \%$; p < 0,001) e de ECAM (7,8\% vs. $24,5 \%$ ). Houve 2 casos de TS definitiva (aos quatro meses e > um ano) no grupo SF e 2 casos de TS possível no grupo SNF. Os índices de morte cardíaca e infarto do miocárdio em dois anos de acompanhamento também foram semelhantes.

Na interpretação dos achados de Mendes et al. ${ }^{7}$, há vários fatores que devem ser considerados. Em primeiro lugar, os índices de sucesso angiográfico para ICP em bifurcações (cerca de 80\%) estão abaixo do padrão atual. Na verdade, no estudo CACTUS ${ }^{8}$ (Coronary Bifurcations: Application of the Crushing Technique Using Sirolimus-Eluting Stents), o sucesso angiográfico foi obtido em 98,5\% dos casos no grupo Crush e em $97,7 \%$ dos casos no grupo Provisional. Os índices de eventos poderiam ter sido ainda menores se os índices de sucesso angiográfico fossem maiores. Em segundo lugar, as bifurcações tratadas envolveram ramos laterais relativamente pequenos $(1,85 \mathrm{~mm} \pm 0,53 \mathrm{~mm}$ com SF e 2,08 mm \pm 0,46 mm com SNF) em comparação aos estudos CACTUS $^{8}$ e Nordic ${ }^{9}$, em que o diâmetro médio dos vasos de referência era de 2,24 mm nos grupos provisionais. É menos provável que ramos laterais menores resultem em angina se uma estenose residual não for tratada ou se ocorrer reestenose, o que pode explicar os baixos índices de RLA vistos em ambos os grupos. Em terceiro lugar, a falta de acompanhamento angiográfico impede qualquer discussão sobre a eficácia anti-reestenótica dos SF ou sobre o local e os padrões da reestenose na bifurcação. Contudo, ela nos permite supor que toda revascularização foi resultante de isquemia. É possível, porém, que um acompanhamento angiográfico seja ainda menos favorável às lesões tratadas com SNF. Finalmente, vale notar que o índice de RLA de 18\% no grupo de SNF foi muito mais baixo que o constatado em estudos históricos sobre SNF em bifurcações (até 36\%) ${ }^{10}$. Do mesmo modo, o índice de RLA no grupo de SF foi extremamente baixo, mas comparável ao índice de $1,9 \%$ no grupo provisional do estudo Nordic ${ }^{9}$. Esse

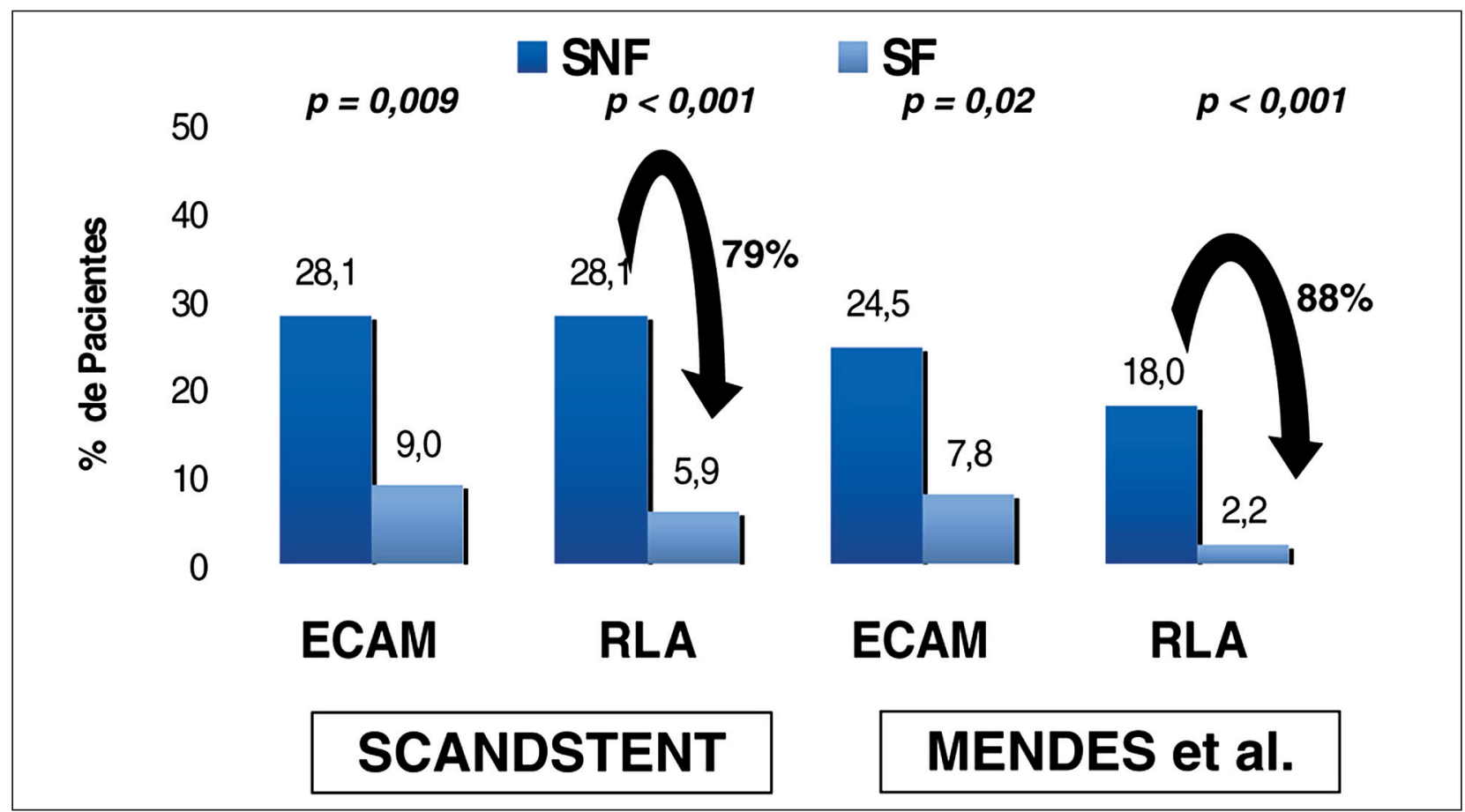

Figura 1 - Eventos cardíacos adversos maiores (ECAM) e índices de revascularização da lesão-alvo (RLA) no estudo SCANDSTENT ${ }^{6}$ e no artigo de Mendes et al. ${ }^{7}$. SNF = stent não-farmacológico; SF = stent farmacológico. 

Superiores aos dos Stents Não-Farmacológicos. Rev Bras Cardiol Invas. 2008;16(4):389-391.

importante achado destaca os excelentes resultados alcançáveis com os SF, principalmente se o acompanhamento angiográfico rotineiro não for realizado.

Concluindo, o estudo atual nos oferece mais evidências sobre a eficácia dos SF em relação aos SNF em bifurcações coronárias. No estudo do "mundo real", reportado por Mendes et al. ${ }^{7}$, os SF foram associados a grande redução da necessidade de nova revascularização, em conformidade com o observado no estudo randomizado SCANDSTENT ${ }^{6}$ (Figura 1). Embora o pequeno tamanho da amostra não permita qualquer conclusão sobre segurança dos SF em bifurcações, é tranqüilizador ver que os índices de óbito e de infarto do miocárdio foram semelhantes nos dois grupos. Em nossa opinião, se não houver contra-indicações para doze meses de terapia antiplaquetária dupla, a estratégia provisional utilizando SF deve ser o tratamento de escolha para ICP em bifurcações que não envolvam o tronco da artéria coronária esquerda.

\section{REFERÊNCIAS BIBLIOGRÁFICAS}

1. Latib A, Colombo A. Bifurcation disease: what do we know, what should we do? J Am Coll Cardiol Intv. 2008;1:218-26.

2. Tsuchida K, Colombo A, Lefevre T, Oldroyd KG, Guetta V, Guagliumi G, et al. The clinical outcome of percutaneous treatment of bifurcation lesions in multivessel coronary artery disease with the sirolimus-eluting stent: insights from the Arterial Revascularization Therapies Study part II (ARTS II). Eur Heart J. 2007;28(4):433-42.

3. Latib A, Cosgrave J, Godino C, Qasim A, Corbett SJ, Tavano $\mathrm{D}$, et al. Sirolimus-eluting and paclitaxel-eluting stents for the treatment of coronary bifurcations. Am Heart J. 2008;156(4): 745-50.
4. lakovou I, Schmidt T, Bonizzoni E, Ge L, Sangiorgi GM, Stankovic G, et al. Incidence, predictors, and outcome of thrombosis after successful implantation of drug-eluting stents. JAMA. 2005;293(17):2126-30.

5. Colombo A, Moses JW, Morice MC, Ludwig J, Holmes DR Jr., Spanos V, et al. Randomized study to evaluate sirolimuseluting stents implanted at coronary bifurcation lesions. Circulation. 2004;109(10):1244-9.

6. Thuesen L, Kelbaek H, Klovgaard L, Helqvist S, Jorgensen E, Aljabbari S, et al. Comparison of sirolimus-eluting and bare metal stents in coronary bifurcation lesions: subgroup analysis of the Stenting Coronary Arteries in Non-Stress/Benestent Disease Trial (SCANDSTENT). Am Heart J. 2006;152(6):1140-5.

7. Mendes A, Sousa AGMR, Costa RA, Moreira A, Costa Jr. JR, Maldonado G, et al. Impacto dos stents farmacológicos no tratamento percutâneo de lesões coronárias em bifurcação: resultados clínicos tardios de um estudo comparativo incluindo pacientes do "mundo-real". Rev Bras Cardiol Invas. $2008 ; 16(4): 406-14$.

8. Colombo A. CACTUS trial (Coronary Bifurcation Application of the Crush Technique Using Sirolimus-Eluting Stents): 6month clinical and angiographic results. In: EuroPCR 2008; Barcelona, Spain. Available at: http://www.europcronline.com/ fo/lecture/view_slide.php?idCongres $=4 \& i d=5899$. Acesso em: 22 de julho de 2008.

9. Steigen TK, Maeng M, Wiseth R, Erglis A, Kumsars I, Narbute l, et al. Randomized study on simple versus complex stenting of coronary artery bifurcation lesions: the Nordic bifurcation study. Circulation. 2006;114(18):1955-61.

10. Yamashita T, Nishida T, Adamian MG, Briguori C, Vaghetti $\mathrm{M}$, Corvaja $\mathrm{N}$, et al. Bifurcation lesions: two stents versus one stent: immediate and follow-up results. J Am Coll Cardiol. 2000;35(5):1145-51

\section{CONFLITO DE INTERESSES}

Antonio Colombo é acionista minoritário da Cappella Inc. (Galway, Irlanda), que fabrica um stent não-farmacológico para lesões em bifurcações. 